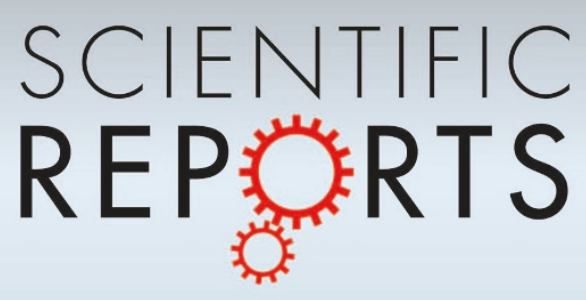

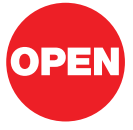

SUBJECT AREAS: ELECTRON TRANSFER

LIGHT HARVESTING

ORGANOCATALYSIS

HOMOGENEOUS CATALYSIS

Received

19 October 2012

Accepted

14 December 2012

Published

11 January 2013

Correspondence and requests for materials should be addressed to X.C.W. (xcwang@fzu. edu.cn)

\section{Ionic Liquid Co-catalyzed Artificial Photosynthesis of $\mathrm{CO}$}

\author{
Jinliang Lin, Zhengxin Ding, Yidong Hou \& Xinchen Wang
}

Research Institute of Photocatalysis, Fujian Provincial Key Laboratory of Photocatalysis-State Key Laboratory Breeding Base, and College of Chemistry and Chemical Engineering, Fuzhou University, Fuzhou 350002, P. R. China.

The conversion of $\mathrm{CO}_{2}$ to chemical feedstocks is of great importance, which yet requires the activation of thermodynamically-stable $\mathrm{CO}_{2}$ by metal catalysts or metalloenzymes. Recently, the development of metal-free organocatalysts for use in $\mathrm{CO}_{2}$ activation under ambient conditions has opened new avenues for carbon fixation chemistry. Here, we report the capture and activation of $\mathrm{CO}_{2}$ by ionic liquids and coupling to photoredox catalysis to synthesize $\mathrm{CO}$. The chemical nature of anions and the organic functional groups on the imidazolium cations of ionic liquids, together with reaction medium have been demonstrated to have remarkable effects on the activation and reduction of $\mathrm{CO}_{2}$. Considering almost unlimited structural variations of ionic liquids by a flexible combination of cations and anions, this photochemical pathway provides unique opportunities for carbon fixation by rationally-designed chemical systems via linking ionic liquid based materials with chromorphoric molecules in tackling the great challenges of artificial photosynthesis.

\begin{abstract}
C onversion of carbon dioxide (a main component of natural photosynthesis) as a renewable $\mathrm{C} 1$ feedstock to value-added compounds (e.g., methane, methanol, carbon monoxide, and sugar) has attracted considerable attention due to its significance in chemical industry, geopolitics and carbon recycling within the ecosystem ${ }^{1-6}$. In nature, the capture, concentration and conversion of atmospheric $\mathrm{CO}_{2}$ is realized by metalloenzymes in photosynthetic organisms such as plants, algae and cyanobacteria that convert $\mathrm{CO}_{2}$, water and solar energy to sugars for the plant and oxygen for Earth's atmosphere. Usually, artificial conversion of extremely-inert $\mathrm{CO}_{2}$ require its catalytic activation by transition-metal catalysts with multiple redox states and subsequently integrating to reduction reactions via multi-electron transfer coupled with protons to avoid high energy inter-
\end{abstract} mediates.

Recent development in the field of $\mathrm{C} 1$ chemistry involves the emergent applications of metal-free organocatalysts, such as frustrated Lewis pairs (FLPs), carbenes, bicyclic amidines, and ionic liquids (ILs) as chemical coordination substrates for the binding and activation of $\mathrm{CO}_{2}$ at room temperature and atmospheric pressure(Fig. 1) ${ }^{7-10}$. For example, FLPs were illustrated to catalyze $\mathrm{CO}_{2}$ reduction to methanol and methane $e^{11}$. $\mathrm{N}$-heterocyclic carbene (NHC) converts $\mathrm{CO}_{2}$ to $\mathrm{CH}_{3} \mathrm{OH}$ via formation of zwitterionic $\mathrm{NHC} \cdot \mathrm{CO}_{2}$ adducts as key intermediates in the reductive deoxygenation of $\mathrm{CO}_{2}$ with diphenylsilane as a stoichiometic reductant ${ }^{12}$. Very recently, Rosen et al. has demonstrated the promoted electrochemical reduction of $\mathrm{CO}_{2}$ to $\mathrm{CO}$ at overpotential of only $0.17 \mathrm{~V}$ by using 1-ethyl-3-methylimidazolium tetrafluoroborate ionic liquid as the $\mathrm{CO}_{2}$ coordinating substrates in water ${ }^{13}$.

ILs are room temperature molten salts, formed by the weak combination of a large organic ion and a chargedelocalized inorganic/organic anion, with versatile structural and functional variations ${ }^{14}$. The scientific and technological importance of ionic liquids is their wide applications in lubricants, electrolytes, catalysts and as gas capture ${ }^{15-17}$. Of particular interest is the promising application of ILs as green solvents with a number of important properties, such as negligible volatility, high stability, high ionic conductivity, high polarity, and solubility with many compounds $\mathrm{s}^{18-22}$.

Room temperature ILs (RTILs) represent a highly versatile and tunable platform for the development of reversible $\mathrm{CO}_{2}$ capture systems with high adsorption capacities. Amine-functionalized task-specific ILs (TSILs) have been demonstrated to show a gravimetric capability of $7 \%\left(0.5 \mathrm{~mol} \mathrm{CO}_{2}\right.$ per mol of the TSIL) for $\mathrm{CO}_{2}$ capture at ambient pressure, but with extreme viscosity that limits kinetics ${ }^{23}$. In addition, the synthesis of amine-functionalized TSILs requires several synthetic and purification steps. Recently, Dai's group developed basic and superbase-derived ILs that showed promise in rapid and switchable $\mathrm{CO}_{2}$ capture with an equimolar absorption capability ${ }^{24}$. The combination of ILs with alkanolamines has also been developed as a viable approach to achieve high levels of reversible $\mathrm{CO}_{2}$ capture in IL solvents ${ }^{25}$. Interestingly, in the above-mentioned carbon 

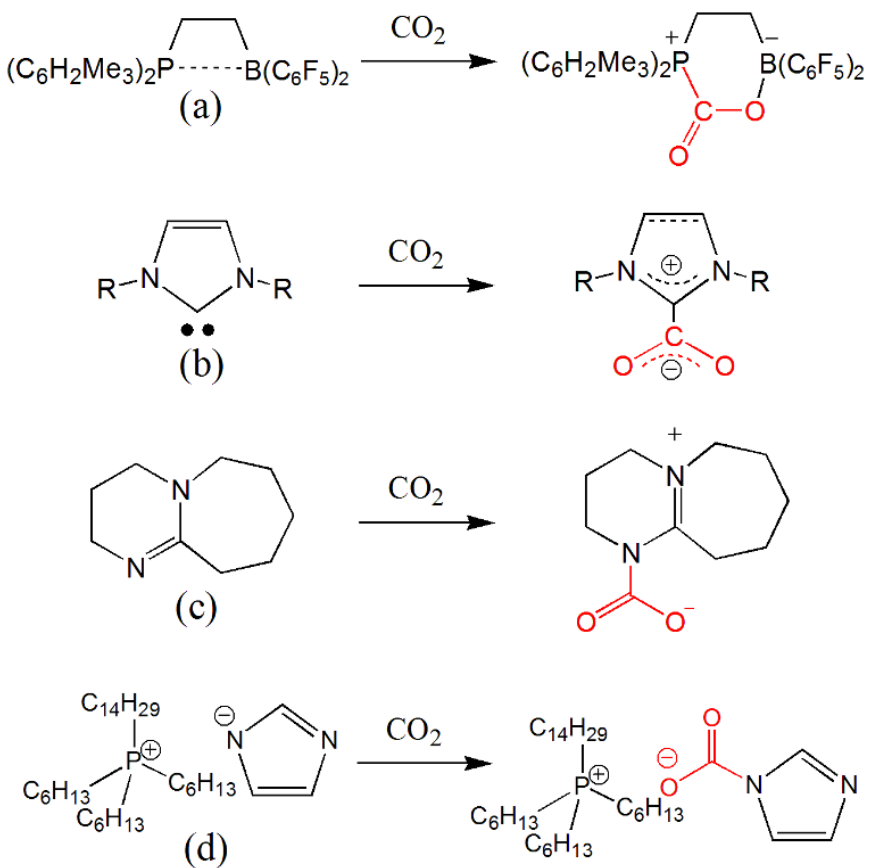

Figure $1 \mid$ Metal-free organocatalysts for $\mathrm{CO}_{2}$ activation by coordinating with frustrated Lewis pair (a), N-heterocyclic carbene (b), bicyclic amidine (c) and ionic liquid (d).

capture systems, the formation of carbamates is evident by binding of $\mathrm{CO}_{2}$ either to the amines tethered to cations (or anions) of ILs or to the amino groups. This way is similar to the first step of plant photosynthetic cycle, in which the $\mathrm{CO}_{2}$ molecule is initially bonded to nitrogen atoms, making reactive carbamate intermediates in the biology system ${ }^{26}$. We are therefore inspired to link the unique coordination chemistry of ILs to artificial photosynthesis for promoting carbon fixation.

Although the uses of ILs to promote $\mathrm{CO}_{2}$ capture and conversion are already evident and has been proposed for future carbon photofixation in high pressure biphasic ILs- $\mathrm{CO}_{2}$ (liquid) systems ${ }^{13,23-25,27,28}$, the merging of IL chemistry with photoredox organocatalysis to achieve gas $\mathrm{CO}_{2}$ fixation with visible light under ambient conditions is rarely covered. Herein, we delineate the application of ILs to facilitate $\mathrm{CO}_{2}$ capture under ambient conditions, and then integrating to a classic photoredox catalytic cycle for efficient $\mathrm{CO}_{2}$ conversion to $\mathrm{CO}^{29}$. This new, IL-promoted $\mathrm{CO}_{2}$ photoconversion protocol was first examined in a photochemical tandem system that contains a catalyst combination of $\left[\mathrm{Ru}(\mathrm{bpy})_{3}\right] \mathrm{Cl}_{2}\left(\mathrm{bpy}=2,2^{\prime}\right.$-bipyridine $)$ and $\mathrm{CoCl}_{2} \cdot 6 \mathrm{H}_{2} \mathrm{O}$ as a light sensitizer and an electron mediator, respectively, along with triethanolamine (TEOA) as an electron donor and a visible light source. The system cooperatively works with ILs to accelerate $\mathrm{CO}_{2}$ photochemical reduction in various solvents at mild conditions.

\section{Results}

First, 1-ethyl-3-methylimidazolium tetrafluoroborate ([EMIM] $\left[\mathrm{BF}_{4}\right]$ ) as a RTIL was introduced into the photocatalytic system that splits $\mathrm{CO}_{2}$ to $\mathrm{CO}$ and [O] in aqueous medium, accompanying by the generation of $\mathrm{H}_{2}$ from the oxidative dehydrogenation of TEOA. Part of the generated $\mathrm{H}_{2}$ (or $2 \mathrm{H}^{+}+2 \mathrm{e}^{-}$) accepted the [O] to form $\mathrm{H}_{2} \mathrm{O}$, and thus closing the two-electron two proton reduction cycle. The sum reaction is $\mathrm{CO}_{2}+2 \mathrm{H}^{+}+2 \mathrm{e}^{-} \rightarrow \mathrm{CO}+\mathrm{H}_{2} \mathrm{O}$. A number of reference experiments were designed to emphasize the role of ILs in the reactions and to optimize the reaction conditions as well. Results are summarized in Table 1. In dark, there is no gas detected in the system. However, when the system was illuminated with visible light,

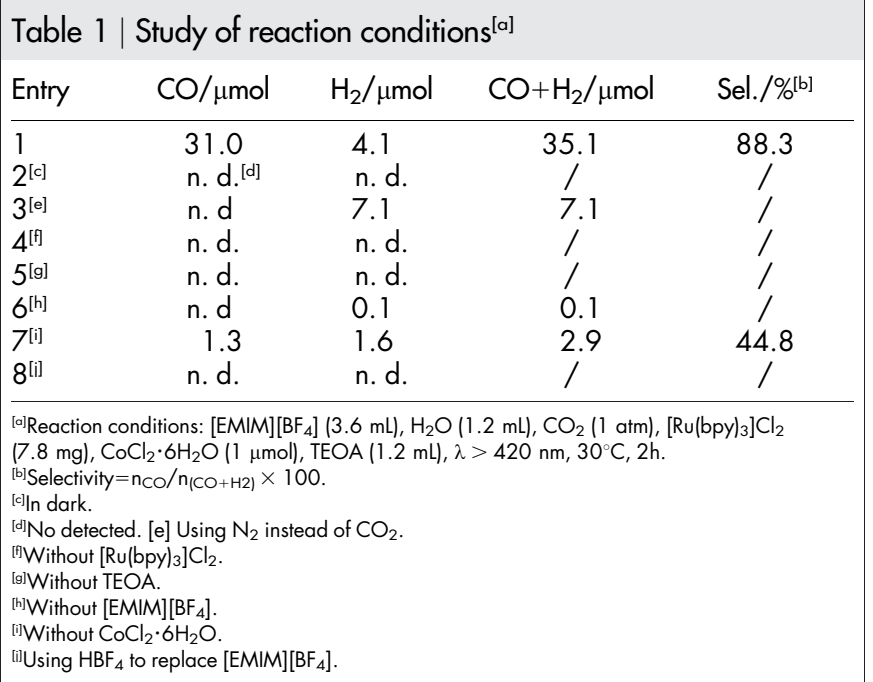

the very stable $\mathrm{CO}_{2}$ molecules were photocatalytically converted into a more reactive $\mathrm{CO}$ species (entry 1 , table 1 ) at a reaction rate of $15.5 \mu \mathrm{mol} / \mathrm{h}$, while releasing $\mathrm{H}_{2}$ at a rate of $2.1 \mu \mathrm{mol} / \mathrm{h}$. These experiments provide strong evidence of the participation of the dye excited state in the catalytic cycle. The mixture of $\mathrm{CO}$ and $\mathrm{H}_{2}$ is the main component of syngas, an important carbon feedstock in chemical industry to make synthetically valuable chemicals (e.g. diesel, methane, methanol, and dimethyl ether $)^{30}$.

Control experiments showed that no reaction occurred in the absence of either $\left[\mathrm{Ru}(\mathrm{bpy})_{3}\right] \mathrm{Cl}_{2}$ or TEOA or [EMIM] $\left[\mathrm{BF}_{4}\right]$ (entry 4-6, table 1). To rule out the potential promotional effect of the $\mathrm{BF}_{4}$ anion (note that $\mathrm{CO}_{2}$ is known to form weak complexes with $\mathrm{BF}_{4}^{-}$ anion), $\mathrm{HBF}_{4}$ was used to replace [EMIM] $\left[\mathrm{BF}_{4}\right]$, and it was found that neither $\mathrm{CO}$ nor $\mathrm{H}_{2}$ could be generated (entry 8 , table 1 ). This is a strong indication that it is the imidazolium cation that significantly influences the reaction process of the $\mathrm{CO}_{2}$ to $\mathrm{CO}$ conversion. When $\mathrm{Co}^{2+}$ as an effective electron transport carrier was absent, as expected, this reaction was restrained dramatically, only yielding a small amount of $\mathrm{CO}$ and $\mathrm{H}_{2}$ (entry 7, table 1).

Once $\mathrm{CO}_{2}$ was replaced by $\mathrm{N}_{2}$ in the system, evidence was observed that confirmed the participation of $\mathrm{CO}_{2}$ in the reaction, because only $\mathrm{H}_{2}$ gas was detected under the reaction conditions, and no $\mathrm{CO}$ was found. Evidently, in the absence of $\mathrm{CO}_{2}$, light-induced electrons reduce proton to produce $\mathrm{H}_{2}$. However, in the presence of $\mathrm{CO}_{2}$ captured by the [EMIM] $\left[\mathrm{BF}_{4}\right]$-TEOA system (termed as $* \mathrm{CO}_{2}$ to differentiate from inert $\mathrm{CO}_{2}$ ), the overall efficiency of the photochemical reduction process increased greatly. Clearly, the photoinduced electrons are kinetically favorable for the reduction of $* \mathrm{CO}_{2}$ over protons under the experimental conditions. As proposed by Bockris and coworkers, a high over-potential is typically needed to convert $\mathrm{CO}_{2}$ since the first step in $\mathrm{CO}_{2}$ conversion is the formation of a $\mathrm{CO}_{2}{ }^{-1}$ intermediate, with a very negative formation potential in water and in most organic solvents ${ }^{31}$. [EMIM] $\left[\mathrm{BF}_{4}\right]$ has been reported to greatly lower the free energy of the formation of the $\mathrm{CO}_{2}{ }^{-}$via complexation (with $\mathrm{EMIM}^{+}$), reducing the overall barrier to the reaction ${ }^{13,32}$. Thus, the IL is favorable not only for $\mathrm{CO}_{2}$ capture but for $\mathrm{CO}_{2}$ activation, acting as a cocatalyst that reduces the potential for formation of the $\mathrm{CO}_{2}{ }^{-}$species.

The role of the $[\mathrm{EMIM}]\left[\mathrm{BF}_{4}\right] / \mathrm{H}_{2} \mathrm{O}$ ratio on the reduction of $\mathrm{CO}_{2}$ was investigated, too. As displayed in the inset of Fig. 2, the production of both $\mathrm{CO}$ and $\mathrm{H}_{2}$ increased with increasing ratio of $[\mathrm{EMIM}]\left[\mathrm{BF}_{4}\right] / \mathrm{H}_{2} \mathrm{O}$ which proved that a high concentration of IL is an important role in the enhancement of photocatalytic activities for $\mathrm{CO}_{2}$ reduction. However, a dramatic decrease in the activity for the $\mathrm{CO}$ and $\mathrm{H}_{2}$ productions was observed when $\mathrm{H}_{2} \mathrm{O}$ was removed. Clearly, the reaction is sluggish to start without $\mathrm{H}_{2} \mathrm{O}$, as both 


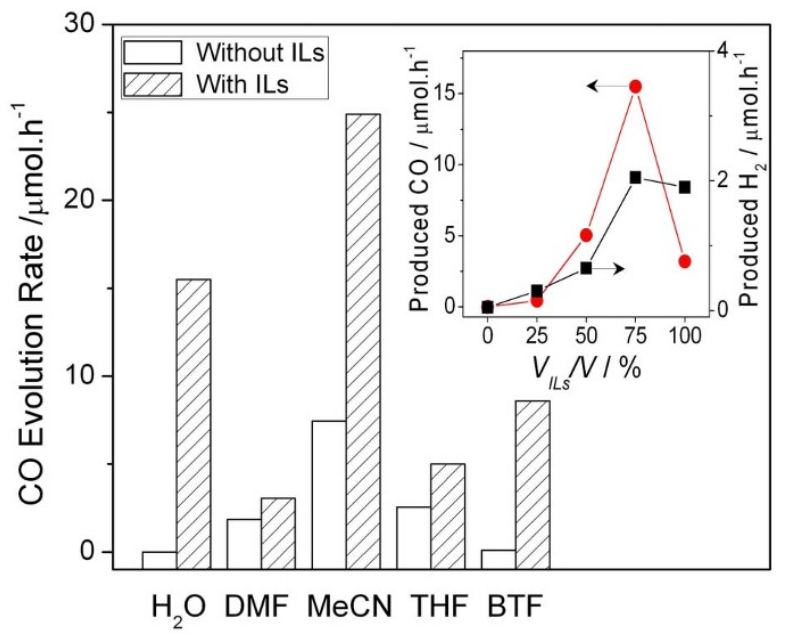

Figure $2 \mid$ The promotional effect of ILs on $\mathrm{CO}_{2}$ photofixation in various solvents. (DMF: $\mathrm{N}, \mathrm{N}$-dimethylformamide. $\mathrm{MeCN}$ : acetonitrile. THF: tetrahydrofufan. BTF: benzenyltrifluoride). The inset is the effect of [EMIM] $\left[\mathrm{BF}_{4}\right] / \mathrm{H}_{2} \mathrm{O}$ ratio on the photocatalytic reduction of $\mathrm{CO}_{2}$.

electrons and holes prefer charged reaction partners. This result indicates that there is a mutual action of IL and $\mathrm{H}_{2} \mathrm{O}$, which plays a critical role on the improvement of photocatalytic activity towards $\mathrm{CO}_{2}$ reduction.

The IL-promoted $\mathrm{CO}_{2}$ photoreduction system was then carried out in various reaction media to determine the generality of the promotional effect of ILs and also to search for a favorable IL-coupler as reaction medium for efficient $\mathrm{CO}_{2}$ photofixation. Various solvents (e.g. DMF, MeCN, THF and BTF) were applied in the $\mathrm{CO}_{2}$ reduction system. The corresponding results are shown in Fig. 2 and Fig. S1. Remarkably, upon adding $[\mathrm{EMIM}]\left[\mathrm{BF}_{4}\right]$, all solvent systems displayed enhanced photocatalytic reactivity towards $\mathrm{CO}_{2}$ reduction, to various extends. In the IL-MeCN mixture, the $\mathrm{CO}$ evolution rate (CER) and the $\mathrm{H}_{2}$ evolution rate (HER) reached 26.3 and $2.1 \mu \mathrm{mol} / \mathrm{h}$, respectively. Notably, in contrast to the generation of $\mathrm{H}_{2}$, the yield of $\mathrm{CO}$ improved much more after the involvement of IL, again reflecting the promoted kinetics of IL for $\mathrm{CO}_{2}$ activation and conversion. Addition of IL was therefore substantially altering selectivity of system towards CO.

As the ultimate objective of artificial photosynthesis is to link $\mathrm{CO}_{2}$ fixation with water splitting, the carbon fixation is desirable to perform in a reaction medium that contains water. The effect of $\mathrm{H}_{2} \mathrm{O}$ on the IL-MeCN system was therefore explored. Results revealed that addition of $15 \% \mathrm{H}_{2} \mathrm{O}$ can significantly increase the CER from 26.3 to $64.0 \mu \mathrm{mol} / \mathrm{h}(\mathrm{TOF}=6.4 / \mathrm{h})$, whereas the best addition amount of water for promoting HER is $8 \%$ (Fig. S2). The addition of water has been reported to have strong effect on the photolabilisation of a bpy ligand from $\left[\mathrm{Ru}(\mathrm{bpy})_{3}\right]^{2+}$ to generate the active catalytic spe$\operatorname{cies}^{33,34}$. Additionally, the reaction rate especially CER agrees with the slightly-enhanced conductivities of water containing media (Table S1). Higher conductivity of electrolyte improves the ability of the fluid environment to support the electron transport during the reduction procedure, which may contribute in part to the enhanced activity. The overall apparent quantum yield of this optimized ILpromoted $\mathrm{CO}_{2}$ photoreduction system was estimated to be $12.3 \%$ under the monochromatic irradiation at $\lambda=420 \mathrm{~nm}$.

Studies on the $\mathrm{CO} / \mathrm{H}_{2}$ evolution as a function of reaction time showed that the relationship between the amount of $\mathrm{CO} / \mathrm{H}_{2}$ produced and the reaction time was non-linear (Fig. 3). After $4 \mathrm{~h}$ illumination, the total production of $\mathrm{CO}$ and $\mathrm{H}_{2}$ reached a maximum value, and thereafter increased slightly. This is consistent with the inherently unstable nature of Ru-based dyes after several turnover numbers in photochemical applications ${ }^{33,34}$. It is therefore

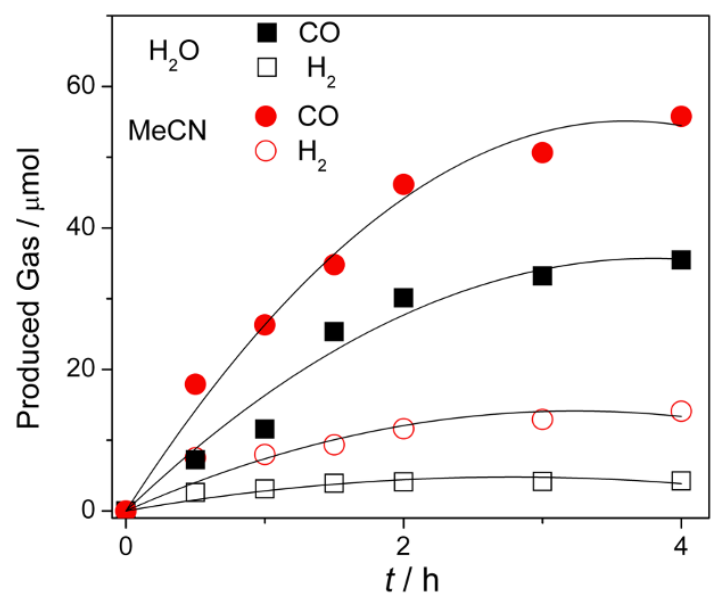

Figure $3 \mid$ The amount of $\mathrm{CO}$ and $\mathrm{H}_{2}$ produced from the IL-promoted $\mathrm{CO}_{2}$ conversion system as a function of reaction time under visible light illumination.

encouraged to use significantly more durable super-molecular and polymeric semiconductors as light energy transducers to couple with ILs for $\mathrm{CO}_{2}$ reduction. Work along this line is in progress in our lab.

\section{Discussion}

The wavelength dependence of $\mathrm{CO}$ evolution revealed that the trend of CO production matched well with the optical absorption spectrum of the antenna molecule (Fig. 4). This investigation provides an extra confirmation that the $\mathrm{CO}_{2}$ reduction process relied on charge photogeneration, separation and the subsequent tandem electron transfer.

Various ILs (shown in Fig. 5) were applied in the reaction system to gain further insights into the effect of the counterions and the substituents of the organic components on the photochemical reduction of $\mathrm{CO}_{2}$. As elucidated in Table 2, the efficiency of $\mathrm{CO}_{2}$ photoreduction was modulated strongly by the different type of counterions examined. The $\mathrm{Tf}_{2} \mathrm{~N}^{-}$anion showed the best promotional effect in the photocatalytic production of $\mathrm{CO}$ and $\mathrm{H}_{2}$ than other anions such as, $\mathrm{L}_{-} \mathrm{L}^{-}, \mathrm{TfO}^{-}, \mathrm{Ac}^{-}, \mathrm{DCA}^{-}$and $\mathrm{BF}_{4}{ }^{-}$. The high structural symmetry of $\mathrm{Tf}_{2} \mathrm{~N}^{-}$anion is known to endow the system with a low viscosity that is favorable for reaction kinetics ${ }^{35}$. Both

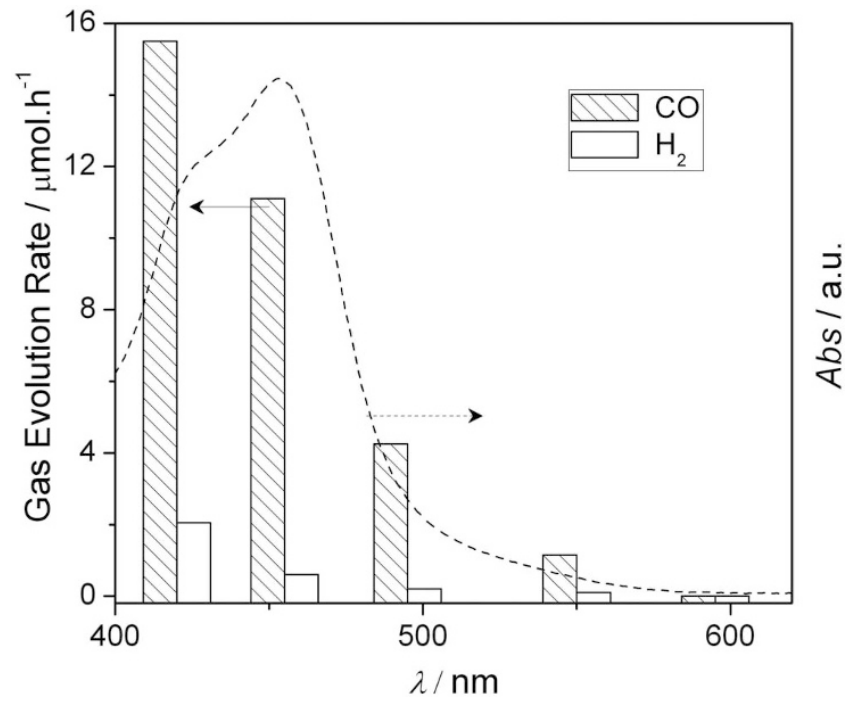

Figure $4 \mid$ The dependence of the wavelength of incident light on the gas evolution rate of $\mathrm{CO}$ and $\mathrm{H}_{2}$ from the $\mathrm{CO}_{2}$ photoreduction system in $[\mathrm{EMIM}]\left[\mathrm{BF}_{4}\right]-\mathrm{H}_{2} \mathrm{O}$ medium. 


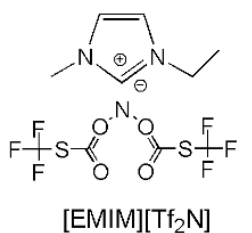

(1)

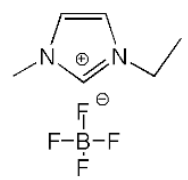

[EMIM][BF 4 ]

(6)

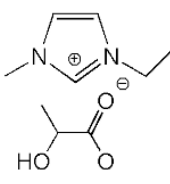

[EMIM][L-L]

(2)

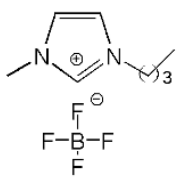

[BMIM] $\left[\mathrm{BF}_{4}\right]$

(7)

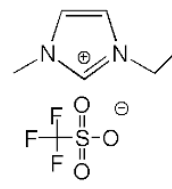

[EMIM][OTf]

(3)

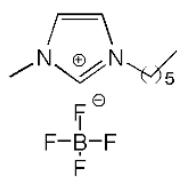

[HMIM][BF $\left.{ }_{4}\right]$

(8)<smiles>CCn1ccn(C)c1=O</smiles>

[EMIM][AC]

(4)

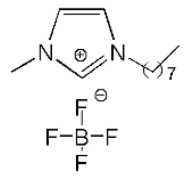

[OMIM] $\left[\mathrm{BF}_{4}\right]$

(9)<smiles>CCn1ccn(C)c1=O</smiles>

[EMIM][DCA]

(5)

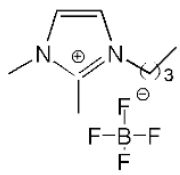

$[\mathrm{BDiMIM}]\left[\mathrm{BF}_{4}\right]$

(10)

Figure $5 \mid$ Chemical structures of various ionic liquids corresponded to those in Table 2 for the photocatalytic conversion of $\mathrm{CO}_{2}$ to $\mathrm{CO}$ under mild conditions.

fluoroalkyl group and $\mathrm{Tf}_{2} \mathrm{~N}^{-}$with a large ionic size are known to increase the interaction of the ILs and $\mathrm{CO}_{2}{ }^{36}$. When the substituted alkyl chain on 1-position of the imidazolium ring was extended from ethyl to octyl, the activities for the production of $\mathrm{CO}$ decreased sequentially. When the $\mathrm{H}$ in the $\mathrm{C}(2)$ position of 7 was substituted with methyl group 10, the activity decreased too. Obviously, the decreased yields were due to the increased length of the carbon chain, associating with the increase in van der Waals interactions and molecular weight. These cause an increased viscosity of the solvent, which together with the increased steric hindrance limits catalytic kinetics, leading to low reactivities ${ }^{36}$. These observations underline the fact that the promotional effect of ILs in the photochemical reduction of $\mathrm{CO}_{2}$ are closely related to both the counterions and the organic functional groups on ILs, which determine the properties of ILs such as viscosity, conductivity, polarity, dielectric constant and acid-base chemistry. Therefore, the wide availability of different cations and anions and their almost unlimited combination has led to a choice of ionic liquids that have a potential synergism between the cation and the anion for supporting $\mathrm{CO}_{2}$ photochemical reduction.

In summary, a combination of $\mathrm{CO}_{2}$ binding and activation by ILs with photoredox catalysis has been developed to achieve the conversion of $\mathrm{CO}_{2}$ to $\mathrm{CO}$ at mild, environmental conditions. The promotional effect of ILs in $\mathrm{CO}_{2}$ photochemcial reduction is strongly related to the chemical properties of counterions and the organic functional groups on the imidazolium cation. IL chemistry thereby continues to facilitate new strategies for the creation of valuable chemicals from $\mathrm{CO}_{2}$ by organocatalysis ${ }^{12}$, here more specifically

Table 2 | The effect of various ILs on the photocatalytic conversion of $\mathrm{CO}_{2}$ to $\mathrm{CO}$ under mild conditions

\begin{tabular}{lcccc} 
IL & $\mathrm{CO} / \mu \mathrm{mol}$ & $\mathrm{H}_{2} / \mu \mathrm{mol}$ & $\mathrm{CO}+\mathrm{H}_{2} / \mu \mathrm{mol}$ & $\mathrm{Sel} . / \%$ \\
\hline (1) & 34.7 & 10.9 & 45.6 & 76.1 \\
$(\mathbf{2})$ & 3.7 & 1.1 & 4.8 & 77.1 \\
(3) & 0.4 & 0.1 & 0.5 & 80.0 \\
$(\mathbf{4})$ & n. d. & n. d. & $/$ & $/$ \\
$(\mathbf{5})$ & 0.1 & 0.2 & 0.3 & 33.3 \\
$(\mathbf{6})$ & 31.0 & 4.1 & 35.1 & 88.3 \\
$(\mathbf{7})$ & 28.0 & 1.6 & 29.6 & 94.6 \\
$(\mathbf{8})$ & 13.1 & 0.5 & 13.6 & 96.3 \\
$(\mathbf{9})$ & 6.8 & 0.3 & 7.1 & 95.8 \\
$(\mathbf{1 0})$ & 13.7 & 1.1 & 14.8 & 92.3 \\
\hline
\end{tabular}

[a] Reaction conditions are the same as those in the Table 1. photoredox organocatalysis. This new photochemical cascade reaction offers a new protocol to split and convert chemically inert $\mathrm{CO}_{2}$, by the extension of IL chemistry to artificial photosynthesis.

\section{Methods}

Chemicals. All the solvents including N,N-dimethylformamide (DMF, anhydrous, 99.8\%), Tetrahydrofufan (THF, anhydrous, 99.9\% ), Acetonitrile (MeCN, anhydrous, $99.8 \%$ ) and Benzenyltrifluoride (BTF, anhydrous, $\geq 99 \%$ ) are purchased from China Sinopharm Chemical reagent $\mathrm{Co}$. and stored over molecular sieve, which were used directly without further purification. Tris(2,2'-bipyridyl)ruthenium(II) chloride hexahydrate (TBR, $>98.0 \%$ ) purchased from Tokyo Chemical Industry Co. Ionic liquids are also commercially supplied ( $\geq 98 \%$ Shyfhx Co.). Other reagents used were analysis grades without further purification.

$\mathrm{CO}_{2}$ photoreduction studies. All experiments were performed in a Schlenk flask $(80 \mathrm{ml})$ under an atmosphere pressure of $\mathrm{CO}_{2}(1 \mathrm{~atm})$. In the Schlenk flask, Tris (2, 2 '-bipyridyl) ruthenium(II) chloride hexahydrate (10 $\mu \mathrm{mol}), \mathrm{CoCl}_{2} \cdot 6 \mathrm{H}_{2} \mathrm{O}(1 \mu \mathrm{mol})$ were dissolved in $6 \mathrm{ml}$ mixture of solvent/ILs/TEOA ( $3: 1: 1$ by volume). This mixture system was subjected to vacuum degassing and backfilling with pure $\mathrm{CO}_{2}$ gas. This process is repeated (three times) and after the last cycle the flask is backfilled with $\mathrm{CO}_{2}$. Then the system was irradiated with a non-focus $300 \mathrm{~W}$ Xe-lamp with a $420 \mathrm{~nm}$ cut-off filter under vigorous stirring at $30^{\circ} \mathrm{C}$ as controlled by a water-cooling system. The produced gases $\left(\mathrm{CO}, \mathrm{H}_{2}\right)$ were detected using gas chromatography (Shimadzu $8 \mathrm{~A}$ ) equipped with a packed molecular sieve column (TDX-1 mesh 42/10). Ar was used as the carrier gas of the GC.

IR characterization. The measured solution was obtained by saturating [EMIM] $\left[\mathrm{BF}_{4}\right]$ with $\mathrm{CO}_{2}$. IR spectra were measured by sandwiching a thin film of the solution between two $\mathrm{CaF}_{2}$ substrates by a Nicolet Magna 670 FT-IR spectrometer. Pure $[\mathrm{EMIM}]\left[\mathrm{BF}_{4}\right]$ is used as reference sample.

Conductivity measurements. The conductivity was determined with a conductivity meter produced by Shanghai Precision \& Scientific Instrument Co. (DDSJ-318) that is equipped with DJS-1C conducting electrodes. The instrument was calibrated with $\mathrm{KCl}$ solution. In the experiment, the solution and the electrode were sealed in a glass tube, which was placed in a constant temperature water bath $(298 \pm 0.05 \mathrm{~K})$. Each measurement was repeated three times and the average values were calculated.

Quantum yield measurements. The apparent quantum yield (AQY) for $\mathrm{CO} / \mathrm{H}_{2}$ generation was measured using the same photochemical experimental setup, but with a LED lamp (low-power $420 \mathrm{~nm}$-LED, $3 \mathrm{~W}$, Shenzhen LAMPLIC Science Co. China) as a light source. The intensity of LED irradiation was measured as $20.6 \mathrm{~mW} / \mathrm{cm}^{2}$ (Newport 842-PE) and the irradiated area was controlled at $1.0 \mathrm{~cm}^{2}$.

1. Thampi, K. R., Kiwi, J. \& Grätzel, M. Methanation and photo-methanation of carbon dioxide at room temperature and atmospheric-press. Nature 327, 506-508 (1987).

2. Arakawa, H. et al. Catalysis research of relevance to carbon management: Progress, challenges, and opportunities. Chem. Rev. 101, 953-996 (2001)

3. Greenbaum, E., Lee, J. W., Tevault, C. V., Blankinship, S. L. \& Mets, L. J. $\mathrm{CO}_{2}$ fixation and photoevolution of $\mathrm{H}_{2}$ and $\mathrm{O}_{2}$ in a mutant of chlamydomonas lacking photosynthesis-I. Nature 376, 438-441 (1995).

4. Lewis, N. S. \& Nocera, D. G. Powering the planet: Chemical challenges in solar energy utilization. Proc. Natl. Acad. Sci. USA 103, 15729-15735 (2006). 
5. Chueh, W. C. et al. High-flux solar-driven thermochemical dissociation of $\mathrm{CO}_{2}$ and $\mathrm{H}_{2} \mathrm{O}$ using nonstoichiometric ceria. Science 330, 1797-1801 (2010).

6. Angamuthu, R., Byers, P., Lutz, M., Spek, A. L. \& Bouwman, E. Electrocatalytic $\mathrm{CO}_{2}$ conversion to oxalate by a copper complex. Science, 327, 313-315 (2010).

7. Mömming, C. M. et al. Reversible metal-free carbon dioxide binding by frustrated Lewis pairs. Angew. Chem. Int. Ed. 48, 6643-6646 (2009).

8. Zhou, H., Zhang, W. Z., Liu, C. H., Qu, J. P. \& Lu, X. B. $\mathrm{CO}_{2}$ adducts of Nheterocyclic carbenes: Thermal stability and catalytic activity toward the coupling of $\mathrm{CO}_{2}$ with epoxides. J. Org. Chem. 73, 8039-8044 (2008).

9. Perez, E. R. et al. Activation of carbon dioxide by bicyclic amidines. J. Org. Chem. 69, 8005-8011 (2004).

10. Voutchkova, A. M., Feliz, M., Clot, E., Eisenstein, O. \& Crabtree, R. H Imidazolium carboxylates as versatile and selective $\mathrm{N}$-heterocyclic carbene transfer agents: Synthesis, mechanism, and applications. J. Am. Chem. Soc. 129, 12834-12846 (2007).

11. Ashley, A. E., Thompson, A. L. \& O'Hare, D. Non-metal-mediated homogeneous hydrogenation of $\mathrm{CO}_{2}$ to $\mathrm{CH}_{3} \mathrm{OH}$. Angew. Chem. Int. Ed. 48, 9839-9843 (2009).

12. Riduan, S. N., Zhang, Y. G. \& Ying, J. Y. Conversion of carbon dioxide into methanol with silanes over N-heterocyclic carbene catalysts. Angew. Chem. Int. Ed. 48, 3322-3325 (2009).

13. Rosen, B. A. et al. Ionic liquid-mediated selective conversion of $\mathrm{CO}_{2}$ to $\mathrm{CO}$ at low overpotentials. Science 334, 643-644 (2011).

14. Wasserscheid, P. Chemistry - Volatile times for ionic liquids. Nature 439, 797-797 (2006).

15. Armand, M., Endres, F., MacFarlane, D. R., Ohno, H. \& Scrosati, B. Ionic-liquid materials for the electrochemical challenges of the future. Nat. Mater. 8, 621-629 (2009).

16. Snyder, J., Fujita, T., Chen, M. W. \& Erlebacher, J. Oxygen reduction in nanoporous metal-ionic liquid composite electrocatalysts. Nat. Mater. 11, 904-907 (2011).

17. Wang, X. Q. \& Dai, S. Ionic liquids as versatile precursors for functionalized porous carbon and carbon-oxide composite materials by confined carbonization. Angew. Chem. Int. Ed. 49, 6664-6668 (2010).

18. Zhao, H. B., Holladay, J. E., Brown, H. \& Zhang, Z. C. Metal chlorides in ionic liquid solvents convert sugars to 5-hydroxymethylfurfural. Science 316, 1597-1600 (2007).

19. Earle, M. J. et al. The distillation and volatility of ionic liquids. Nature 439, 831-834 (2006).

20. Cooper, E. R. et al. Ionic liquids and eutectic mixtures as solvent and template in synthesis of zeolite analogues. Nature 430, 1012-1016 (2004).

21. Rogers, R. D. \& Seddon, K. R. Ionic liquids - Solvents of the future? Science $\mathbf{3 0 2}$ 792-793 (2003).

22. Welton, T. Room-temperature ionic liquids. Solvents for synthesis and catalysis. Chem. Rev. 99, 2071-2083 (1999).

23. Bates, E. D., Mayton, R. D., Ntai, I. \& Davis, J. H. $\mathrm{CO}_{2}$ capture by a task-specific ionic liquid. J. Am. Chem. Soc. 124, 926-927 (2002).

24. Wang, C. M., Luo, H. M., Jiang, D. E., Li, H. R. \& Dai, S. Carbon Dioxide Capture by Superbase-Derived Protic Ionic Liquids. Angew. Chem. Int. Ed. 49, 5979-5981 (2010).

25. Gurkan, B. et al. Molecular design of high capacity, low viscosity, chemically tunable ionic liquids for $\mathrm{CO}_{2}$ capture. J. Phys. Chem. Lett. 1, 3494-3499 (2010).

26. Cleland, W. W., Andrews, T. J., Gutteridge, S., Hartman, F. C. \& Lorimer, G. H. Mechanism of Rubisco: The carbamate as general base. Chem. Rev. 98, 549-561 (1998).

27. Zhang, Z. F. et al. Hydrogenation of carbon dioxide is promoted by a task-specific ionic liquid. Angew. Chem. Int. Ed. 47, 1127-1129 (2008).
28. Grills, D. \& Fujita, E. New Directions for the Photocatalytic reduction of $\mathrm{CO}_{2}$ : Supramolecular, $\operatorname{scCO}(2)$ or biphasic ionic liquid-scCO(2) systems. J. Phys. Chem. Lett. 1, 2709-2718 (2010).

29. Lehn, J. M. \& Ziessel, R. Photochemical generation of carbon monoxide and hydrogen by reduction of carbon dioxide and water under visible light irradiation. Proc. Natl. Acad. Sci. USA 79, 701-704 (1982).

30. Spath, P. L. \& Dayton, N. D. C. REL/TP-510-34929. National Renewable Energy Laboratory: Golden, CO, 2003.

31. Bockris, J. O. M. \& Wass, J. C. The photoelectrocatalytic reduction of carbon dioxide. J. Electrochem. Soc. 136, 2521 (1989).

32. Rosen, B. A. et al. In situ spectroscopic examination of a low overpotential pathway for carbon dioxide conversion to carbon monoxide. J. Phys. Chem. C 116, 15307-15312 (2012).

33. Ziessel, R., Hawecker, J. \& Lehn, J. M. Photogeneration of carbon monoxide and of hydrogen via simultaneous photochemical reduction carbon dioxide and water by visible light irradiation of organic solutions containing tris(2,2'-bipyridine) Ruthenium (II) and cobalt (II) species as homogeneous catalysts. Helv Chim Acta. 69, 1065-1084 (1986).

34. Lehn, J. M. \& Ziessel, R. Photochemical reduction of carbon dioxide to formate catalyzed by 2,2'-bipyridine-Ruthenium(II) or 1,10-phenanthrolineRuthenium(II) complexes. J. Organometallic Chem. 382, 157-173 (1990)

35. Baltus, R. E., Culbertson, B. H., Dai, S., Luo, H. \& DePaoli, D. W. Low-pressure solubility of carbon dioxide in room-temperature ionic liquids measured with a quartz crystal microbalance. J. Phys. Chem. B 108, 721-727 (2004).

36. Almantariotis, D., Gefflaut, T., Padua, A. A. H., Coxam, J. Y. \& Gomes, M. F. C. Effect of fluorination and size of the alkyl side-chain on the solubility of carbon dioxide in 1-alkyl-3-methylimidazolium bis(trifluoromethylsulfonyl)amide ionic liquids. J. Phys. Chem. B 114, 3608-3617 (2010)

\section{Acknowledgements}

This work was financially supported by the National Basic Research Program of China (Grant No. 2013CB632405) and the National Natural Science Foundation of China (Grant Nos. 21033003 and 21173043). We also thank the Department of Education of Fujian Province in China for funding.

\section{Author contributions}

X.W. planed and supervised the project. J.L. conducted the photocatalytic performances. Z.D. and Y.H. commented on the manuscript writing and the result discussion. All authors contributed to data analysis and writing of this manuscript.

\section{Additional information}

Supplementary information accompanies this paper at http://www.nature.com/ scientificreports

Competing financial interests: The authors declare no competing financial interests.

License: This work is licensed under a Creative Common Attribution-NonCommercial-NoDerivs 3.0 Unported License. To view a copy of this license, visit http://creativecommons.org/licenses/by-nc-nd/3.0/

How to cite this article: Lin, J., Ding, Z., Hou, Y. \& Wang, X. Ionic Liquid Co-catalyzed Artificial Photosynthesis of CO. Sci. Rep. 3, 1056; DOI:10.1038/srep01056 (2013). 